\title{
Situação epidemiológica da brucelose bovina no Estado de Sergipe
}

\author{
[Epidemiological situation of bovine brucellosis in the State of Sergipe, Brazil] \\ V.G.S.O. Silva ${ }^{1}$, R.A. Dias ${ }^{1}$, F. Ferreira ${ }^{1}$, M. Amaku ${ }^{1}$, E.L.S. Costa ${ }^{2}$, J.R. Lôbo ${ }^{3}$, \\ V.C.F. Figueiredo ${ }^{3}$, V.S.P. Gonçalves ${ }^{4}$, J.S. Ferreira Neto ${ }^{1 *}$ \\ ${ }^{1}$ Faculdade de Medicina Veterinária e Zootecnia - USP \\ Av. Prof. Dr. Orlando Marques de Paiva, 87 \\ 05508-270 - São Paulo, SP \\ ${ }^{2}$ Delegacia Federal de Agricultura de Sergipe - Aracaju, SE \\ ${ }^{3}$ Departamento de Saúde Animal - SDA-MAPA - Brasília, DF \\ ${ }^{4}$ Faculdade de Agronomia e Medicina Veterinária - UnB - Brasília, DF
}

\begin{abstract}
RESUMO
Realizou-se um estudo para caracterizar a situação epidemiológica da brucelose bovina no Estado de Sergipe. O Estado foi estratificado em dois circuitos produtores. Em cada circuito produtor foram amostradas aleatoriamente cerca de 300 propriedades e, dentro dessas foi escolhido de forma aleatória um número pré-estabelecido de animais, dos quais foi obtida uma amostra de sangue. No total foram amostrados 4.757 animais, provenientes de 590 propriedades. Em cada propriedade amostrada foi aplicado um questionário epidemiológico para verificar o tipo de exploração da propriedade e as práticas zootécnicas e sanitárias que poderiam estar associadas ao risco de infecção pela doença. O protocolo de testes utilizado foi o da triagem com o teste do antígeno acidificado tamponado e a confirmação dos positivos com o teste do 2-mercaptoetanol. O rebanho foi considerado positivo, se pelo menos um animal foi reagente às duas provas sorológicas. A prevalência de focos e a de animais foram: 12,6\% [9,2-16,0\%] e 3,4\% [2,3-4,4\%], respectivamente. As prevalências de focos e de animais infectados para os circuitos pecuários foram: circuito $1,11,1 \%$ [7,9-15,0\%] e 2,6\% [1,6-3,5\%]; circuito 2, 12,9\% [9,1-17,6\%] e $6,2 \%$ [3,0-9,5\%]. Os fatores de risco (odds ratio, OR) associados à condição de foco foram: assistência veterinária $(\mathrm{OR}=2,89[1,15-7,23])$, tamanho do rebanho $\geq 30$ fêmeas adultas $(\mathrm{OR}=1,88$ [1,07-3,28]) e uso de inseminação artificial ( $\mathrm{OR}=1,92[0,84-4,38])$.
\end{abstract}

Palavras-chave: bovino, brucelose, prevalência, fatores de risco, Sergipe

\begin{abstract}
A study to characterize the epidemiological situation of bovine brucellosis in the State of Sergipe was carried out. The State was divided in two regions. Around three hundred herds were randomly sampled in each region and a pre-established number of animals were sampled in each of these herds. A total of 4,757 serum samples from 590 herds were collected. In each herd, it was applied an epidemiological questionnaire focused on herd traits as well as husbandry and sanitary practices that could be associated with the risk of infection. The serum samples were screened by the Rose-Bengal Test (RBT) and positive sera were re-tested by the 2-mercaptoethanol test (2-ME). The herd was considered positive if at least one animal was positive on both RBT and 2-ME tests. The prevalences of infected herds and animals in the State were, respectively: $12.6 \%$ [9.2-16.0\%] and 3.4\% [2.3-4.4\%]. The prevalences of infected herds and animals in the regions were, respectively: region 1, 11.1\% [7.9-15.0\%] and 2.6\% [1.6-3.5\%]; and region 2, 12.9\% [9.1-17.6\%] and 6.2\% [3.0-9.5\%]. The risk factors associated with the presence of the infection were: veterinary assistance $(O R=2.89$ [1.15-7.23]), herd size $\geq 30$ adult females $(O R=1.88$ [1.07-3.28]), and use of artificial insemination (OR=1.92 [0.84-4.38]).
\end{abstract}

Keywords: cattle, brucellosis, prevalence, risk factors, Sergipe, Brazil

Recebido em 27 de março de 2009

Aceito em 23 de setembro de 2009

*Autor para correspondência (corresponding author)

E-mail: jsoares@vps.fmvz.usp.br 


\section{INTRODUÇÃO}

O Estado de Sergipe, localizado na região Nordeste no Brasil, ocupa a superfície de $22.005 \mathrm{~km}^{2}$, correspondendo a $0,26 \%$ do território nacional e é constituído por 75 municípios. Tem cerca de 1 milhão de cabeças de bovinos (IBGE, 2007).

Os principais responsáveis pela importância da pecuária do Estado foram os grandes produtores de bovinos de corte, com animais importados do Estado da Bahia para o centro-sul do Estado e também para as regiões que margeiam o Rio São Francisco (Dezen, 2007; DEAGRO-SE; comunicação pessoal).

A bovinocultura de corte desenvolveu-se na região da Cotinguiba, que abrange os municípios de Carmópolis, Divina Pastora, Japaratuba, Laranjeiras, Maruim, Nossa Senhora do Socorro, Rosário do Catete, Santa Rosa e Siriri. Também merecem destaque os municípios de Lagarto, Riachão do Dantas, Simão Dias, Pinhão, Pedra Mole, Aquidabã, Própria, Frei Paulo, Carira, Nossa Senhora das Dores, Cumbe, Feira Nova e Ribeirópolis. A produção de carne bovina é feita de forma extensiva, com pouco uso de inseminação artificial ou transferência de embriões. As raças mais utilizadas são a Nelore, a Tabapuã e seus mestiços (Sergipe, 2007).

A bovinocultura de leite desenvolveu-se na região do semiárido, sendo o município de Nossa Senhora da Glória o maior produtor estadual. Atualmente, o rebanho leiteiro é predominantemente do tipo misto (carne e leite), com tendência à especialização leiteira. O sistema de produção varia de extensivo a semiintensivo. A maior parte da produção concentrase nas mãos de pequenos produtores que se dedicam à fabricação de queijos. A indústria queijeira trouxe desenvolvimento e melhora das condições de vida aos sertanejos da região, no entanto a maioria apresenta problemas de natureza sanitária e funciona de forma clandestina (Sergipe, 2007).

A primeira instituição a tratar do assunto agrário em Sergipe surgiu em 1915, por meio do Decreto $\mathrm{n}^{\mathrm{o}}$ 608, que criou a Diretoria de Obras, Agricultura, Indústria e Aviação. Em 1991, após várias reformas administrativas, a Empresa de Assistência Técnica e Extensão Rural
(EMATER) passou a ser denominada Empresa de Desenvolvimento Agropecuário de Sergipe (EMDAGRO), responsável pelas atividades de pesquisa agropecuária, sanidade animal e vegetal. A partir de 2004, a denominação passou a ser Departamento de Desenvolvimento Agropecuário de Sergipe (DEAGRO/SE) (Sergipe, 2007).

Os primeiros registros de brucelose bovina no Estado ocorreram a partir de 1975, com o início do Programa Nacional de Sanidade Animal (PRONASA). Na maioria das vezes o registro foi a de suspeita clínica seguida de confirmação por exames sorológicos específicos (Dezen, 2007; DEAGRO-SE; comunicação pessoal). Nesse mesmo ano, o Ministério da Agricultura realizou o primeiro grande estudo de brucelose bovina no País, envolvendo 18 Estados. Sergipe foi incluído nesse estudo e apresentou uma prevalência nos animais de 10,5\%. O teste utilizado foi o da soroaglutinação rápida em placa (Brasil, 1977).

Em 1983, a Embrapa-Sergipe realizou um estudo visando conhecer a situação das doenças da esfera reprodutiva no rebanho do Estado, bem como verificar a eficiência do Card Test para o diagnóstico da brucelose bovina. Foram detectados $6,8 \%$ de animais positivos ao Card Test, $5,8 \%$ de animais positivos e $11,0 \%$ de suspeitos no teste de soroaglutinação rápida em placa.

Assim, como a brucelose ainda não está adequadamente caracterizada em todo Estado, o presente estudo teve por objetivos estimar a prevalência e identificar os fatores de risco para a brucelose bovina, e fornecer subsídios para a melhor implementação e gestão do Programa Nacional de Controle e Erradicação da Brucelose e Tuberculose (PNCEBT) em Sergipe.

\section{MATERIAL E MÉTODOS}

O estudo foi planejado por técnicos do Ministério da Agricultura, Pecuária e Abastecimento, da Universidade de São Paulo e da Universidade de Brasília, e o trabalho de campo foi executado pelo DEAGRO-SE no período de outubro de 2002 a dezembro de 2003.

Para o conhecimento das diferenças regionais nos parâmetros epidemiológicos da brucelose 
bovina, o Estado foi dividido em dois circuitos produtores de bovinos, levando-se em consideração os diferentes sistemas de produção, práticas de manejo, finalidades de exploração, tamanho médio de rebanhos e sistemas de comercialização. Essa divisão em regiões produtoras também levou em conta a capacidade operacional e logística do serviço veterinário oficial do Estado para a realização das atividades de campo, baseando-se nas áreas de atuação das suas unidades regionais.

Em cada circuito produtor, estimou-se a prevalência de propriedades infectadas pela brucelose bovina e a de animais soropositivos por meio de um estudo amostral em dois estágios, dirigido para detectar focos da doença. No primeiro estágio, sorteou-se, aleatoriamente, um número pré-estabelecido de propriedades com atividade reprodutiva (unidades primárias de amostragem). No segundo, sorteou-se um número pré-estabelecido de fêmeas bovinas com idade igual ou superior a 24 meses (unidades secundárias de amostragem).

Nas propriedades rurais onde existia mais de um rebanho, foi escolhido o rebanho bovino de maior importância econômica, no qual os animais estavam submetidos ao mesmo manejo, ou seja, sob os mesmos fatores de risco. A escolha da unidade primária de amostragem foi aleatória, baseada no cadastro de propriedades rurais com atividade reprodutiva de bovinos. A propriedade sorteada que, por motivos vários, não pôde ser visitada, foi substituída por outra, nas proximidades e com as mesmas características de produção. O número de propriedades selecionadas por circuito foi estimado pela fórmula para amostras simples aleatórias (Thrusfield, 2007). Os parâmetros adotados para o cálculo foram: nível de confiança de 0,95 , prevalência estimada de 0,25 e erro de 0,05. A capacidade operacional e financeira do serviço veterinário oficial do Estado também foi levada em consideração para a determinação do tamanho da amostra por circuito.

O planejamento amostral para as unidades secundárias visou estimar um número mínimo de animais a serem examinados dentro de cada propriedade de forma a permitir a sua classificação como foco ou não foco de brucelose. Para tanto, foi utilizado o conceito de sensibilidade e especificidade agregadas (Dohoo et al., 2003). Para efeito dos cálculos foram adotados os valores de $95 \%$ e $99,5 \%$, respectivamente, para a sensibilidade e a especificidade do protocolo de testes utilizado (Fletcher et al., 1998) e $20 \%$ para a prevalência estimada. Nesse processo foi utilizado o programa Herdacc versão 3 , e o tamanho da amostra escolhido foi aquele que permitiu valores de sensibilidade e especificidade de rebanho iguais ou superiores a $90 \%$. Assim, nas propriedades com até 99 fêmeas com idade superior a 24 meses, foram amostrados 10 animais e nas com 100 ou mais fêmeas com idade superior a 24 meses, 15 animais. A escolha das fêmeas dentro das propriedades foi casual sistemática.

O protocolo do sorodiagnóstico foi composto pela triagem com o teste do antígeno acidificado tamponado (Rosa Bengala), seguida do reteste dos positivos com o teste do 2-mercaptoetanol, de acordo com as recomendações do PNCEBT (Brasil, 2006). O sangue foi coletado por punção da veia jugular com agulha descartável estéril em tubo com vácuo, previamente identificado. Os soros, armazenados em microtubos de plástico, foram mantidos a $-20^{\circ} \mathrm{C}$ até a realização dos testes. Os testes sorológicos foram realizados no laboratório do DEAGRO/SE.

A propriedade foi considerada positiva quando se detectou pelo menos um animal positivo. As propriedades que apresentaram animais com resultado sorológico inconclusivo, sem nenhum positivo, foram classificadas como suspeitas e excluídas das análises. O mesmo tratamento foi dado aos animais com resultados sorológicos inconclusivos.

O planejamento amostral permitiu determinar as prevalências de focos e de fêmeas adultas $(\geq 24 \mathrm{~m})$ soropositivas para brucelose no Estado e também nos circuitos produtores. Os cálculos das prevalências aparentes e os respectivos intervalos de confiança foram realizados conforme preconizado por Dean et al. (1994). Os cálculos das prevalências de focos e de animais no Estado, e de prevalências de animais dentro das 
regiões foram feitos de forma ponderada (Dohoo et al., 2003).

O peso de cada propriedade no cálculo da prevalência de focos no Estado foi dado por

$$
P_{1}=\frac{\text { propriedades na região }}{\text { propriedades amostradas na região }}
$$

O peso de cada animal no cálculo da prevalência de animais no Estado foi dado por

$P_{2}=\frac{\text { fêmeas } \geq 24 \text { meses na propriedade }}{\text { fêmeas } \geq 24 \text { meses amostradas na propriedade }} \times \frac{\text { fêmeas } \geq 24 \text { meses na região }}{\text { fêmeas } \geq 24 \text { meses nas propriedades amostradas na região }}$
$\mathrm{Na}$ expressão acima, o primeiro termo refere-se ao peso de cada animal no cálculo das prevalências de animais dentro das regiões.

Em cada propriedade amostrada, além da colheita de sangue para a sorologia, foi também aplicado o questionário epidemiológico, elaborado para obter informações sobre o tipo de exploração e as práticas de manejo empregadas, de forma a permitir a realização do estudo de fatores de risco associados à presença da brucelose.

As variáveis analisadas foram: tipo ou sistema de exploração (carne, leite e misto), tipo de criação (confinado, semiconfinado, extensivo), uso de inseminação artificial, raças predominantes, número de vacas com idade superior a 24 meses, número de bovinos na propriedade, presença de outras espécies domésticas, presença de animais silvestres, destino da placenta e dos fetos abortados, compra e venda de animais, vacinação contra brucelose, abate de animais na propriedade, aluguel de pastos, pastos comuns com outras propriedades, pastos alagados, piquete de parição e assistência veterinária.

As variáveis foram organizadas de modo a apresentarem-se em escala crescente de risco. Quando necessário, realizou-se a recategorização dessas variáveis. A categoria de menor risco foi considerada como base para a comparação das demais categorias. As variáveis quantitativas foram categorizadas em percentis.

Foi feita uma primeira análise exploratória dos dados (univariada) para seleção daquelas com $\mathrm{p} \leq 0,20$ para o teste do $\chi^{2}$ ou exato de Fisher e, subsequente, oferecimento dessas à regressão logística. Os cálculos foram realizados com o auxílio do programa SPSS, versão 9.0.
Todas as informações geradas pelo trabalho de campo e de laboratório foram inseridas em um banco de dados específico, utilizado nas análises epidemiológicas.

\section{RESULTADOS E DISCUSSÃO}

O Estado foi dividido em dois circuitos (Fig. 1). Os dados censitários, tomados como base para os cálculos da amostra, foram os mais atualizados e disponíveis à época do trabalho de campo, em 2003. A Tab. 1 traz um resumo desses dados e da amostra estudada em cada um dos circuitos produtores.

Em 14 propriedades do circuito 1, os animais positivos ao teste do antígeno acidificado tamponado não foram submetidos à confirmação pelo teste do 2-mercaptoetanol. Essas propriedades foram consideradas focos. $\mathrm{O}$ mesmo fato ocorreu com 22 propriedades do circuito 2. Todos os 49 animais positivos ao teste do antígeno acidificado tamponado que não foram submetidos ao teste confirmatório foram considerados como infectados e mantidos no cálculo de prevalência de animais.

Embora existissem bubalinos no Estado no momento dos trabalhos de campo e os animais tenham sido selecionados aleatoriamente para a amostragem, nenhum bubalino foi sorteado para participar da amostra.

$\mathrm{Na}$ Tab. 2, mostram-se os resultados de prevalência de focos no Estado e nos circuitos produtores. A Tab. 3 traz a prevalência de focos por tipo de exploração da propriedade e a Tab. 4 , a prevalência de animais.

$\mathrm{Na}$ Tab. 5 mostram-se os resultados da análise univariada e na Tab. 6, o modelo final da regressão logística. 


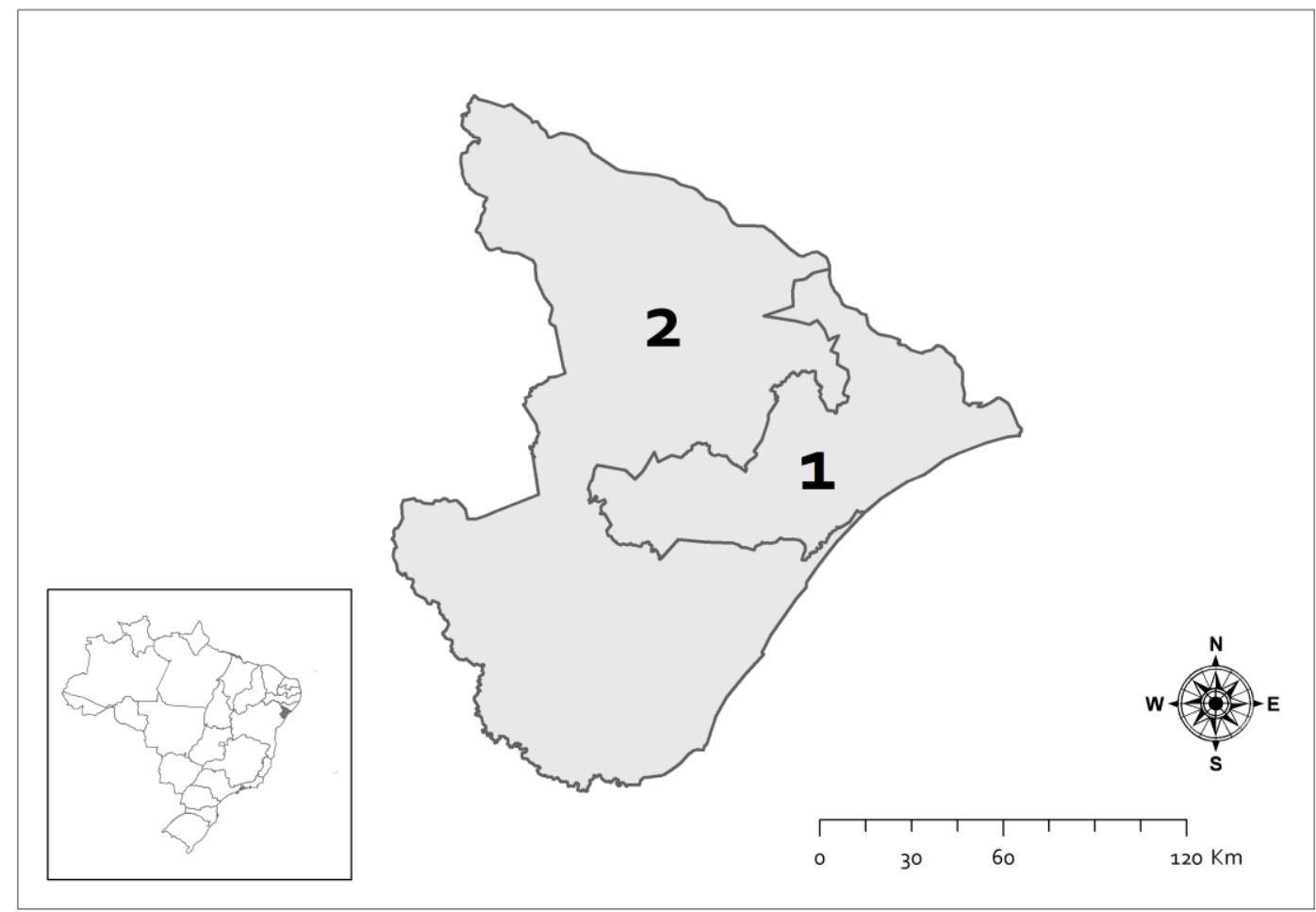

Figura 1. Mapa do Estado de Sergipe com representação dos circuitos pecuários. No detalhe, a localização do Estado de Sergipe no Brasil.

Tabela 1. Dados censitários da população bovina do Estado de Sergipe em 2001, segundo o circuito produtor

\begin{tabular}{rccccc}
\multirow{2}{*}{$\begin{array}{c}\text { Circuito } \\
\text { produtor }\end{array}$} & \multicolumn{2}{c}{$\begin{array}{c}\text { Propriedades com atividade } \\
\text { reprodutiva }\end{array}$} & & \multicolumn{2}{c}{ Fêmeas com idade $\geq 24$ meses } \\
\cline { 2 - 3 } \cline { 5 - 5 } & total & amostradas & & total & amostradas \\
\hline 1 & 3.582 & 328 & & 262.442 & 2.620 \\
2 & 17.022 & 263 & & 70.473 & 2.137 \\
Total & 20.604 & 591 & 322.915 & 4.757 \\
\hline
\end{tabular}

Fonte: Sergipe, 2007.

Tabela 2. Prevalência de focos de brucelose bovina na propriedade, segundo o circuito produtor, no Estado de Sergipe

\begin{tabular}{|c|c|c|c|c|}
\hline \multirow{2}{*}{ Circuito produtor } & \multicolumn{2}{|c|}{ Propriedades } & \multirow{2}{*}{ Prevalência (\%) } & \multirow{2}{*}{ IC $(95 \%)$} \\
\hline & Testadas & Positivas & & \\
\hline 1 & 325 & 36 & 11,07 & {$[7,87-15,00]$} \\
\hline 2 & 263 & 34 & 12,92 & {$[9,12-17,59]$} \\
\hline Total & 588 & 70 & 12,60 & {$[9,19-16,01]$} \\
\hline
\end{tabular}

IC: intervalo de confiança. 
Tabela 3. Prevalência (Prev) de focos de brucelose bovina extratificado por tipo de exploração, segundo o circuito produtor, no Estado de Sergipe

\begin{tabular}{|c|c|c|c|c|c|c|}
\hline \multirow{2}{*}{$\begin{array}{l}\text { Circuito } \\
\text { produtor }\end{array}$} & \multicolumn{2}{|c|}{ Corte } & \multicolumn{2}{|c|}{ Leite } & \multicolumn{2}{|c|}{ Misto } \\
\hline & Prev (\%) & IC $(95 \%)$ & Prev (\%) & IC (95\%) & Prev (\%) & IC (95\%) \\
\hline 1 & 7,14 & {$[0,87-23,50]$} & 14,74 & {$[9,58-21,29]$} & 7,80 & {$[3,95-13,53]$} \\
\hline 2 & 9,52 & {$[2,65-22,62]$} & 13,40 & {$[7,33-21,82]$} & 13,70 & {$[8,19-21,04]$} \\
\hline
\end{tabular}

IC: intervalo de confiança.

Tabela 4. Prevalência de bovinos sororreagentes para brucelose, segundo o circuito produtor, no Estado de Sergipe, 2003

\begin{tabular}{cccccc}
\hline \multirow{2}{*}{ Circuito produtor } & \multicolumn{3}{c}{ Animais } & \multirow{2}{*}{ Prevalência $(\%)$} & IC $(95 \%)$ \\
\cline { 2 - 5 } & 1 & Testados & Positivos & 2,58 & {$[1,62-3,54]$} \\
\multirow{2}{*}{2} & 2.573 & 63 & 6,25 & {$[3,00-9,49]$} \\
Total & 2.067 & 71 & 3,36 & {$[2,28-4,44]$} \\
\hline
\end{tabular}

IC: intervalo de confiança.

Tabela 5. Resultados da análise univariada dos possíveis fatores de risco para brucelose bovina em rebanhos com atividade reprodutiva no Estado de Sergipe, 2003

\begin{tabular}{|c|c|c|c|}
\hline Variável & $\begin{array}{c}\text { Propriedades } \\
\text { expostas/propriedades } \\
\text { foco } \\
\end{array}$ & $\begin{array}{c}\text { Propriedades } \\
\text { expostas/propriedades } \\
\text { não foco } \\
\end{array}$ & $\mathrm{p}$ \\
\hline Presença de ovinos e caprinos & $25 / 70$ & $162 / 518$ & 0,541 \\
\hline Presença de equídeos & $68 / 70$ & $455 / 518$ & 0,033 \\
\hline Presença de suínos & $17 / 70$ & $92 / 517$ & 0,251 \\
\hline Presença de aves & $43 / 70$ & $297 / 518$ & 0,602 \\
\hline Presença de cão & $45 / 70$ & $351 / 518$ & 0,656 \\
\hline Presença de gato & $37 / 70$ & $237 / 518$ & 0,322 \\
\hline Presença de animais silvestres & $27 / 70$ & $177 / 518$ & 0,554 \\
\hline Presença de cervídeos & $1 / 70$ & $4 / 518$ & $0,471 *$ \\
\hline Presença de capivaras & $0 / 70$ & $3 / 518$ & $1,0 *$ \\
\hline Presença de outros silvestres & $27 / 70$ & $175 / 518$ & 0,511 \\
\hline Comprar machos e fêmeas para reprodução & $39 / 70$ & $294 / 515$ & 0,929 \\
\hline Alugar pasto em alguma época do ano & $12 / 70$ & $152 / 517$ & 0,045 \\
\hline Utilizar pasto em comum com outras propriedades & $4 / 70$ & $78 / 518$ & 0,053 \\
\hline Presença de áreas alagadiças na propriedade & $27 / 70$ & $132 / 518$ & 0,03 \\
\hline Criação confinada ou semi confinada & $36 / 70$ & $203 / 518$ & 0,068 \\
\hline Ocorrência de aborto nos últimos 12 meses & $15 / 69$ & $79 / 500$ & 0,284 \\
\hline Ter assistência veterinária & $8 / 70$ & $16 / 518$ & $0,004 *$ \\
\hline Não realizar teste de brucelose & $56 / 70$ & $450 / 518$ & 0,169 \\
\hline Não utilizar piquete de parição & $46 / 70$ & $380 / 516$ & 0,21 \\
\hline Utilizar inseminação artificial & $10 / 70$ & $30 / 518$ & $0,019 *$ \\
\hline Destino impróprio para material do aborto & $29 / 42$ & $230 / 313$ & 0,673 \\
\hline $\begin{array}{l}\text { Não vacinar ou vacinar fêmeas em qualquer idade } \\
\text { com a B19 }\end{array}$ & $64 / 70$ & $491 / 516$ & $0,247 *$ \\
\hline $\begin{array}{l}\text { Ter } \geq 30 \text { fêmeas com idade igual ou superior a } 24 \\
\text { meses }\end{array}$ & $31 / 68$ & $123 / 513$ & 0 \\
\hline
\end{tabular}

*Corrigido pelo teste exato de Fisher. 
Situação epidemiológica da brucelose bovina no Estado de Sergipe

Tabela 6. Modelo final da regressão logística multivariada de fatores de risco (odds ratio) para brucelose bovina em rebanhos com atividade reprodutiva no Estado de Sergipe

\begin{tabular}{lccc}
\multicolumn{1}{c}{ Variável } & Odds ratio & IC (95\%) & $\mathrm{p}$ \\
\hline Ter $\geq 30$ fêmeas com idade $\geq 24$ meses & 1,88 & {$[1,07-3,28]$} & 0,026 \\
Ter assistência veterinária & 2,89 & {$[1,15-7,23]$} & 0,023 \\
Utilizar inseminação artificial & 1,92 & {$[0,84-4,38]$} & 0,117 \\
\hline
\end{tabular}

IC: intervalo de confiança.

A prevalência de focos de brucelose bovina no Estado foi de $12,6 \%$ (Tab. 2). Os dados também mostram que os valores de prevalência de focos nos circuitos são semelhantes.

Na Tabela 3, embora os valores pontuais de prevalência de focos sejam diferentes para as propriedades de tipo corte, leite e misto, não há diferença entre as propriedades nos dois circuitos.

No circuito 1, formado principalmente por rebanhos de corte (Sergipe, 2007), os resultados de prevalência de focos para as propriedades do tipo leite e misto foram, respectivamente, de $14,7 \%$ e $7,8 \%$ (Tab. 3). Esse circuito abrange a região do vale do rio Cotinguiba, onde, em 1980, Pedreira et al. (1983) examinaram amostras de soro sanguíneo de 3.597 bovinos dos municípios de Capela, Japaratuba, Rosário do Catete, Siriri, Maruim, Riachuelo e Laranjeiras e classificaram $53,1 \%$ das propriedades que produziam leite como focos de brucelose. Esses autores utilizaram a soroaglutinação rápida em placa e o Card Test e examinaram 50\% das propriedades com atividade leiteira. Dentro da propriedade, amostraram $50 \%$ das matrizes e o total de machos reprodutores existentes. Os autores não informaram se os resultados dos testes foram interpretados em série, em paralelo ou individualmente.

A prevalência de animais soropositivos à brucelose no Estado foi de 3,4\% (Tab. 4), bem menor do que a verificada pelo Ministério da Agricultura em 1975 (10,5\%; Brasil, 1977). Os dados também mostram que os valores de prevalência de animais nos circuitos são iguais, embora os valores pontuais sejam muito diferentes (Tab. 4). A prevalência de animais encontrada no circuito 1 (2,5\%; Tab. 4) foi inferior aos 6,8\% verificados por Pedreira et al.
(1983), em rebanhos leiteiros da região do vale do rio Cotinguiba. Em resumo, a situação da brucelose no Estado é homogênea em relação aos circuitos e ao tipo de exploração da unidade de criação.

A comparação dos resultados do presente estudo com aqueles realizados em 1975 (Brasil, 1977) e 1980 (Pedreira et al., 1983), em que pese a diferença de metodologia, tanto em relação ao protocolo de testes quanto ao delineamento amostral, sugere que a prevalência da doença tem diminuído com o passar dos anos, provavelmente como resultado da melhora de práticas sanitárias adotadas pela cadeia produtora de carne e leite. Entretanto, a prevalência de $12,6 \%$ de focos e a de 3,4\% de animais (Tab. 2 e 4) podem ser consideradas altas para uma doença de difusão lenta como a brucelose. A diminuição dessa prevalência pode ser conseguida mediante aumento da cobertura vacinal com a amostra B19.

Considerando apenas os valores pontuais, o fato de o circuito 2 apresentar prevalência de animais superior ao circuito 1 (Tab. 4) sugere que a prevalência de animais soropositivos intrarebanho no circuito 2 seja maior do que no circuito 1. Embora as Tab. 2 e 4 mostrem diferenças nos valores pontuais de prevalência para os dois circuitos, o delineamento adotado não permitiu constatar diferenças.

Em relação ao tipo de exploração (Tab. 3), a doença está homogeneamente distribuída nos rebanhos de corte e leite, em ambos os circuitos. Assim, a brucelose bovina está homogeneamente distribuída nos dois circuitos do Estado de Sergipe e também nas propriedades do tipo leite, carne e misto. 
O modelo final da regressão logística apontou como fatores de risco as variáveis assistência veterinária e tamanho do rebanho ( $\geq 30$ vacas). $\mathrm{O}$ uso da inseminação artificial, embora não tenha sido estatisticamente significante, foi mantido no modelo final como variável de ajuste, pois melhorou sua capacidade preditiva e mostrou um valor de $\mathrm{P}$ mais alto que 0,1 (Tab. 6).

Parece paradoxal que o fato de haver assistência veterinária signifique aumento de risco para a brucelose bovina. $\mathrm{O}$ que, porém, provavelmente ocorreu no Estado é o hábito de contratar serviços veterinários só após a constatação de transtornos reprodutivos, comumente associados à infecção brucélica. Assim, a assistência veterinária é uma consequência, não uma causa da presença da doença.

Quanto ao tamanho do rebanho, as propriedades com 30 ou mais vacas com idade igual ou superior a 24 meses (segundo tercil) tiveram 1,88 vezes mais chances de serem focos de brucelose do que as com número menor de fêmeas (Tab. 6). Isso significa que a brucelose é mais frequente em grandes propriedades. A associação entre tamanho do rebanho e presença de brucelose foi demonstrada em vários estudos (Kellar et al., 1976; Nicoletti 1980; Salman e Meyer, 1984). Nos grandes rebanhos não há diferenças individuais quanto à suscetibilidade à doença, mas algumas características desses grandes rebanhos podem facilitar a transmissão da brucelose, tais como: maior frequência de reposição de animais, maior quantidade de problemas relacionados ao controle sanitário e influência na dinâmica da doença (Crawford et al., 1990). Christie (1969) observou que o aumento do rebanho resulta em aumento da probabilidade de ocorrer a doença e de persistir a infecção, e aumento da prevalência da doença e da dificuldade de erradicá-la. Assim, número elevado de animais no rebanho significa maior risco de introdução da brucelose pela compra mais frequente de reprodutores. É importante enfatizar que a introdução de animais significa risco real apenas quando praticada sem cuidados sanitários. Assim, ser propriedade de corte ou mista no Estado significa ter mais animais e introduzir reprodutores com mais frequência. Esse resultado sugere, portanto, de forma indireta, que a introdução de reprodutores é a prática que está associada à condição de foco da brucelose.
A inseminação artificial, quando praticada com sêmen de touros infectados é uma forma bastante eficiente de difundir a brucelose bovina, pois o sêmen é depositado diretamente no interior do útero, produzindo infecção com doses muito baixas de desafio (Acha e Szyfres, 2001; Paulin e Ferreira Neto, 2003). Assim, o verdadeiro problema é a prática de inseminação artificial sem cuidados sanitários, ou seja, sem a garantia de que o doador seja livre da infecção. O Estado deve alertar os criadores sobre os riscos envolvidos na utilização da inseminação artificial sem cuidados sanitários e na introdução de animais sem a realização de testes.

Recomenda-se: concentrar esforços na obtenção, em todos os anos, de uma cobertura vacinal mínima de $80 \%$ de fêmeas entre três e oito meses de idade com a vacina B19; desestimular a utilização de sêmen proveniente de centrais de inseminação não certificadas pelo MAPA e a introdução de animais sem a realização de testes para brucelose.

\section{AGRADECIMENTOS}

À FAPESP, ao CNPq, ao DEAGRO-SE e ao MAPA pelo apoio financeiro.

\section{REFERÊNCIAS BIBLIOGRÁFICAS}

ACHA, P.N.; SZYFRES, B. Zoonosis y enfermedades transmisibles comunes al hombre y a los animales: Bacterioses e micoses 3.ed. Washington: OPAS, 2001. 416p

BRASIL. Ministério da Agricultura e Abastecimento. Diagnóstico de saúde animal. Brasília, 1977. 735p.

BRASIL. Ministério de Agricultura, Pecuária e Abastecimento. Programa Nacional de Controle e Erradicação da Brucelose e da Tuberculose Animal (PNCEBT): Manual técnico. Brasília, 2006. $184 \mathrm{p}$.

CRAWFORD, R.P.; HUBER, J.D.; ADAMS, B.S. Epidemiology and surveillance. In: NIELSEN, K.; DUNCAN, J.R. (Ed.). Animal brucellosis. Boca Raton: CRC Press, 1990. p.131-151.

CHRISTIE, T.E. Eradication of brucellosis in northern Ireland: Field problems and experiences. Vet. Rec., v.85, p.268-269, 1969. 
DEAN, A.G.; DEAN, J.A.; COLOMBIER, D. et al. Epi-Info, version 6: A word processing database and statistics program for epidemiology on microcomputers. Atlanta: CDC, 1994. 601p.

DOHOO, I.; MARTIN, W.; STRYHN, H. Veterinary epidemiologic research. Charlottetown, Canadá: Atlantic Veterinary College, 2003. 706p.

FLETCHER, R.H.; FLETCHER, S.W.; WAGNER, E.H. Clinical epidemiology: The essentials. 2.ed. Baltimore: Williams \& Wilkins, 1998. 246p.

IBGE. Disponível em: <http://www.ibge.gov.br/estadosat/temas.php?sig la $=$ se \&tema=pecuaria2006 $>$ Acessado em: 7 mai. 2007.

KELLAR, J.; MARRA, R.; MARTIN, W. Brucellosis in Ontario: A case control study. Can. J. Comp. Med., v.40, p.119-128, 1976.

NICOLETTI, P. The epidemiology of bovine brucellosis. Adv. Vet. Sci. Comp. Med., v.24, p.69-98, 1980.
PAULIN, L.M.; FERREIRA-NETO, J.S. $O$ combate à brucelose bovina: Situação brasileira. Jaboticabal: FUNEP, 2003. 154p.

PEDREIRA, P.A.S.; OLIVEIRA, A.A.; ALMEIDA, M.F.R.S. Índice de ocorrência de brucelose bovina no Vale do Cotinguiba em Sergipe. Comun. Tec. Embrapa, n.7, 4p., 1983.

SALMAN, M.D.; MEYER, M.E. Epidemiology of bovine brucellosis in the Mexicali Valley, México: Literature review of disease-associated factors. Am. J. Vet. Res., v.45, p.1557-1560, 1984.

SERGIPE. Secretaria de Estado da Agricultura e do Desenvolvimento Agrário. Disponível em: $<$ http://www.sagri.se.gov.br/modules/tinyd0/inde x.php?id=47>. Acessado em: 8 ago. 2007.

THRUSFIELD, M. Veterinary epidemiology. 3.ed. Oxford: Blackwell Science, 2007. 610p. 